\title{
The effect of cortical thickness and thread profile dimensions on stress and strain in bone-anchored implants for amputation prostheses
}

This paper was downloaded from TechRxiv (https://www.techrxiv.org).

\section{LICENSE}

CC BY 4.0

SUBMISSION DATE / POSTED DATE

$28-04-2021 / 30-04-2021$

\section{CITATION}

Thesleff, Alexander; Ortiz-Catalan, Max; Brånemark, Rickard (2021): The effect of cortical thickness and thread profile dimensions on stress and strain in bone-anchored implants for amputation prostheses. TechRxiv. Preprint. https://doi.org/10.36227/techrxiv.14499759.v1

$\mathrm{DOI}$

10.36227/techrxiv.14499759.v1 
Title

The effect of cortical thickness and thread profile dimensions on stress and strain in bone-anchored implants for amputation prostheses

\section{Authors}

Alexander Thesleff ${ }^{1,2,3}$, Max Ortiz-Catalán ${ }^{1,2,4,5}$, Rickard Brånemark ${ }^{5,6}$

1. Center for Bionics and Pain Research, Mölndal, Sweden

2. Dept. of Electrical Engineering, Chalmers University of Technology, Gothenburg, Sweden

3. Integrum AB, Mölndal, Sweden

4. Operational Area 3, Sahlgrenska University Hospital, Mölndal, Sweden

5. Department of Orthopaedics, Institute of Clinical Sciences, Sahlgrenska Academy, University of Gothenburg, Sahlgrenska University Hospital, Gothenburg, Sweden

6. Center for Extreme Bionics, Biomechatronics Group, MIT Media Lab, Massachusetts Institute of Technology, Cambridge, MA, USA

Research supported by the Promobilia Foundation, the IngaBritt and Arne Lundbergs Foundation, the Swedish Foundation for Strategic Research (SSF), the Swedish Innovation Agency (VINNOVA), and the Swedish Research Council (Vetenskapsrådet), and Integrum AB.

\section{Abstract}

Skeletal attachment of limb prostheses ensures load transfer between the prosthetic leg and the skeleton. For individuals with lower limb amputation, these loads may be of substantial magnitude. To optimize the design of such systems, knowledge about the structural interplay between implant design features, dimensional changes, and material properties of the implant and the surrounding bone is needed. Here, we present the results from a parametric finite element investigation on a generic bone-anchored implant system of screw design, exposed to external loads corresponding to average and high ambulatory loading. Of the investigated parameters, cortical thickness had the largest effect on the stress and strain in the bone-anchored implant and in the cortical bone. $36 \%-$ $44 \%$ reductions in maximum longitudinal stress in the bone-anchored implant was observed as a result of increased cortical thickness from $2 \mathrm{~mm}$ to $5 \mathrm{~mm}$. Changes in thread depth had larger effect on the maximum stresses in the fixture and the bone than changes in thread root radius within the evaluated parameter space. Stress reductions in the percutaneous abutment were obtained by autologous transplantation of bone tissue distal to the fixture. Results from this investigation may guide structural design optimization for bone-anchored implant systems for attachment of limb prostheses.

\section{Background}

For individuals with amputation, the socket-stump interface is the source of several commonly reported problems such as pain, pressure sores, skin problems, poor retention, and perspiration [1][6]. To address these issues, a method of bone-anchored attachment of limb prostheses was developed, where a surgically implanted percutaneous skeletal extension allows for direct attachment of the prosthesis [7]. The method builds on the discovery that bone tissue may form an exceptionally intimate and mechanically stable connection with titanium [8]. This phenomenon is now known as osseointegration and is defined as "the firm anchoring of a surgical implant by the growth of bone 
around it without fibrous tissue formation at the interface" [9]. The first clinical applications of osseointegration were in dental field, but it has since then spread to other applications such as hearing aids, facial reconstructions, and orthopaedic applications including bone-anchored attachment of prosthetic limbs [10]. The method of bone-anchored attachment of prosthetic limbs was developed in Sweden with the first successful surgeries performed in 1990 [11]. A standardized implant system and treatment protocol was introduced in 1999 as the OPRA (Osseointegrated Prostheses for the Rehabilitation of Amputees) implant system (Integrum AB, Mölndal, Sweden). The OPRA implant system is CE-marked for transfemoral, transhumeral, and thumb/finger amputations, and custommade implants are available for the aforementioned levels, as well as for transtibial and transradial amputations. The treatment has gained increasing clinical acceptance and is currently available in 12 countries. The OPRA implant system was recently granted a pre-market approval (PMA) by the Food and Drug Administration (FDA) in the USA for treatment at the transfemoral level. Following the successful clinical results of the OPRA implant system a few other systems for bone-anchored attachment of prosthetic limbs have been developed, examples are the ILP (Integral Leg Prosthesis, ESKA Orthopaedic Handels GmbH, Germany) (also known as Endo-Exo Prosthesis (EEP)) and the OPL (Osseointegrated Prosthetic Limb, Permedica s.p.a., Italy). With bone-anchored attachment of the prosthesis, a socket is no longer needed and all aforementioned problems related to socket attachment are eliminated. Research on individuals who have undergone treatment with boneanchored attachment of limb prosthesis shows that it leads to improved quality of life, increase in prosthetic function and use, improved walking ability, walking efficiency, range of motion, sitting comfort, and sensory perception from the prosthesis via a phenomenon known as osseoperception [12]-[21].

The OPRA implant system is a modular system, of which the structural components are the fixture and the abutment. The fixture is a cylindrical, externally threaded component, fully implanted into the residual bone. The abutment, which is the percutaneous component has its proximal end press-fitted into the fixture, and its distal end protruding out from the skin and allowing for attachment of the prosthesis. The rigid connection between the prosthesis and the skeleton leads to a more efficient gait compared with the more compliant socket connection [12], [19]. However, it also means that any loads applied to the prosthesis are transferred directly to the bone-anchored implant and the periprosthetic bone which could be at risk of fracture. Several studies involving load cell measurements at the implant prosthesis interface have offered insights in the magnitude of the loading during ambulatory activities of daily living [22]-[24]. This data is valuable for numerical simulations such as finite element (FE) analyses to calculate periprosthetic and implant stress and strain.

The objective of this study was to perform a parametric evaluation on the effect of thread profile parameters and cortical thickness, on the peak stress and strains in a generic implant model subjected to external loads corresponding to average and high ambulatory loading. An additional aim was to quantify effects of changes in bone stiffness characteristics and distal bone resorption on the maximum stresses in the implant system. The generic model was inspired by the OPRA implant system; an externally threaded fixture and other components manufactured from medical grade titanium (Ti6Al4V). The intention was to provide knowledge on how the careful selection of design features can minimize the risk of bone or implant fracture during use. This study contrasts earlier FEbased studies which have been either case studies with subject specific anatomy [25]-[32], studies focused exclusively on bone remodelling around the implant [31], [33], or FE-implementations with too low resolution to accurately capture the stress in the threaded region [25], [27]-[30], [32], [34][37]. 


\section{Method}

The generic model used in this study is shown in Figure 1. The geometrical parameters that we investigated were thread depth (d), the thread root radius $\left(r_{\text {root }}\right)$, and the cortical thickness $(t)$ within the parameter space shown in Figure 1 . The thread was a standard V-shaped thread with $30^{\circ}$ flank angle, a rounded thread crest with constant radius of $0.1 \mathrm{~mm}$ and either a small or a large root radius, of $0.1 \mathrm{~mm}$ or $0.5 \mathrm{~mm}$, respectively. Three thread depths were evaluated from $0.75 \mathrm{~mm}$ to $1.5 \mathrm{~mm}$. The thread pitch $(p)$ was determined by the thread depth, where a larger thread depth led to a larger pitch. It was kept constant for each thread depth, regardless the value of root radius in order to discern the effect of this parameter. Two cortical thicknesses were evaluated, a small cortical thickness of 2 $\mathrm{mm}$ which correspond to the minimum requirement after implantation according to the OPRA surgical protocol [38], and a larger cortical thickness of $5 \mathrm{~mm}$.

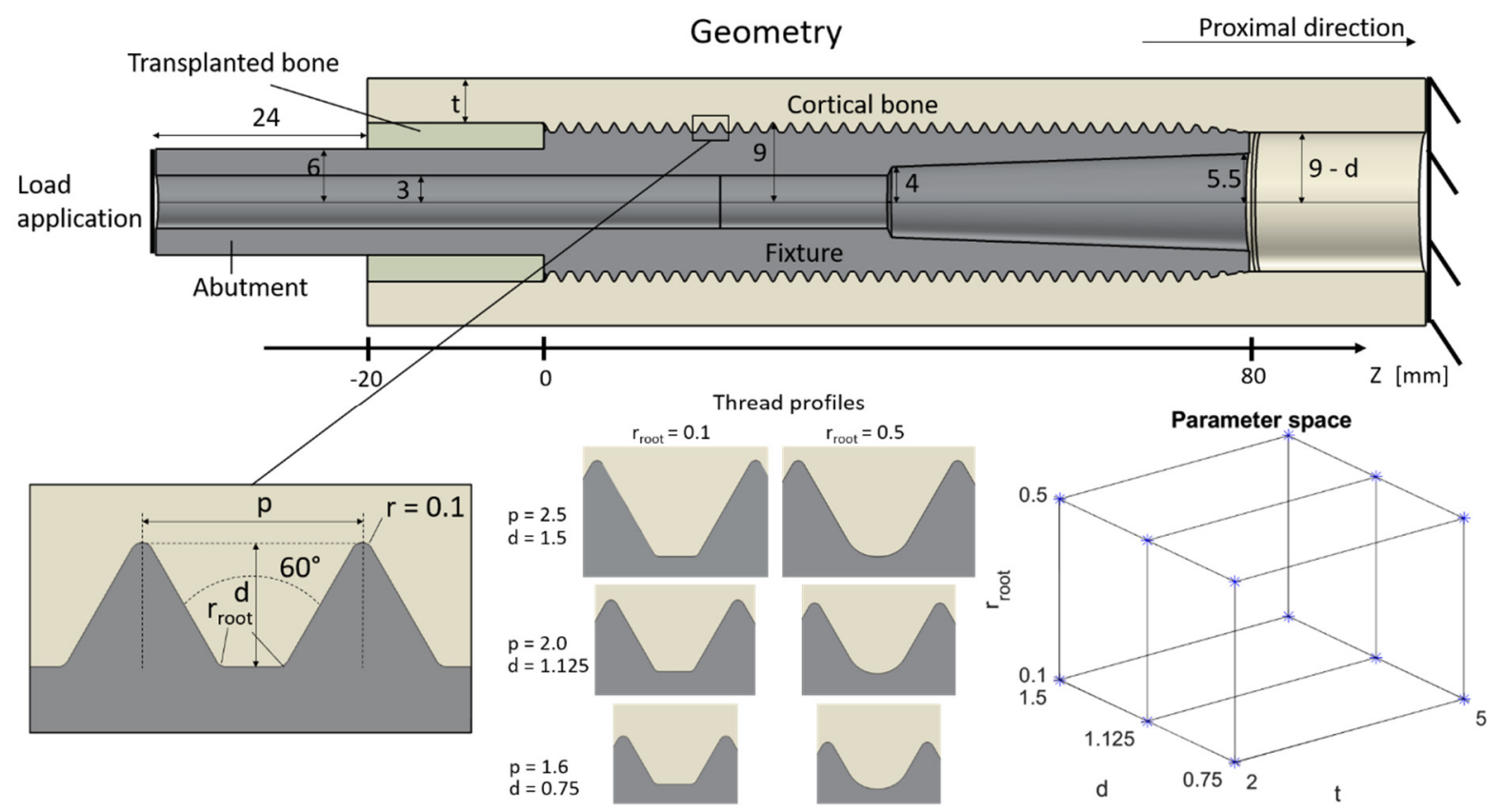

Figure 1. Generic geometry with boundary conditions and dimensions. Dimensions denoted with variable names were varied within the study, whereas dimensions with numerical values were fixed.

\section{Analytical estimation of load sharing}

Prior to performing the FE-simulations, an analytical analysis was performed on a simplified geometry, considering the implant and the cortical bone as two uniform cylinders (without threads) with dimensions as indicated in Figure 1. In this analysis both the Ti6Al4V and the cortical bone were assumed to have isotropic properties with Young's modulus of $110 \mathrm{GPa}$ and $17 \mathrm{GPa}$, respectively. Assuming a pure bending load case, the analytical load sharing between the implant and the cortical bone was calculated based on respective component's bending stiffness, defined as the product of Young's modulus, $(E)$, and the area moment of inertia according, (I), according to Eq. 1, with $\left(d_{0}\right)$ and $\left(d_{i}\right)$ being the external and internal diameter respectively.

$$
E I=E \frac{\pi}{64}\left(d_{o}^{4}-d_{i}^{4}\right)
$$

This initial investigation was conducted with the purpose to serve as a point of reference for the FEsimulation results. 


\section{FE-model}

FE-modelling and simulations were performed using COMSOL Multiphysics version 5.5 (Comsol AB, Sweden). A generic 3D axisymmetric FE-model of the implant system and surrounding bone was created (Figure 1), where the symmetry in geometry allowed for reduction of the model to half of the full geometry. The abutment and fixture were combined into a single component. In accordance with the surgical protocol of the OPRA implant system, the fixture was countersunk $20 \mathrm{~mm}$ into the cortical bone with the distal void filled with a less stiff autologous bone transplant [39], [40]. The interfaces between fixture and the cortical bone and between fixture and transplanted bone were modelled with shared nodes, assuming full osseointegration, (which is close to what has been observed from histological assessment of a retrieved fixture [41]), whereas the interface between the abutment and the transplanted bone was modelled assuming no osseointegration and frictionless sliding. The cortical bone extended $20 \mathrm{~mm}$ distally and proximally from both ends of the fixture. The proximal end of the cortical bone was constrained in all degrees of freedom. The external load was applied at the distal end of the abutment as a static load case.

To meet computational limitations in the hardware while ensuring a sufficiently dense mesh along the full geometry of the threaded implant, a stepwise submodelling scheme was employed (Figure 3). A global model with the full model geometry was created, transversely partitioned at locations indicated in Figure 3. Subsequently, five densely meshed sub-models were created. Global model simulation results (displacements) at the partition surfaces were applied as boundary conditions (prescribed displacement) at the corresponding surfaces in the submodels to generate high resolution simulation results for each of the submodels. Simulation results along longitudinal evaluation profiles in each of the submodels were exported from COMSOL and imported into Matlab (version 2019a, MathWorks Inc., Natick, MA, USA). Custom written scripts were used to integrate the results between the submodels and to visualize the results along the combined evaluation profiles. To avoid non-physical stress singularities at the border of the submodels due to mesh size inconsistencies between the submodels and the global model, a small overlap was included between the neighbouring sub-models (yellow regions in Figure 3). In the data processing stage, the exported simulation results close to the longitudinal end points of the sub-model were discarded so that each submodel only contributed with simulation results starting from the center of the overlapping zone. In addition to eliminating singularities at the submodel borders, this ensured that no duplicate data was used to form the combined simulation results. 


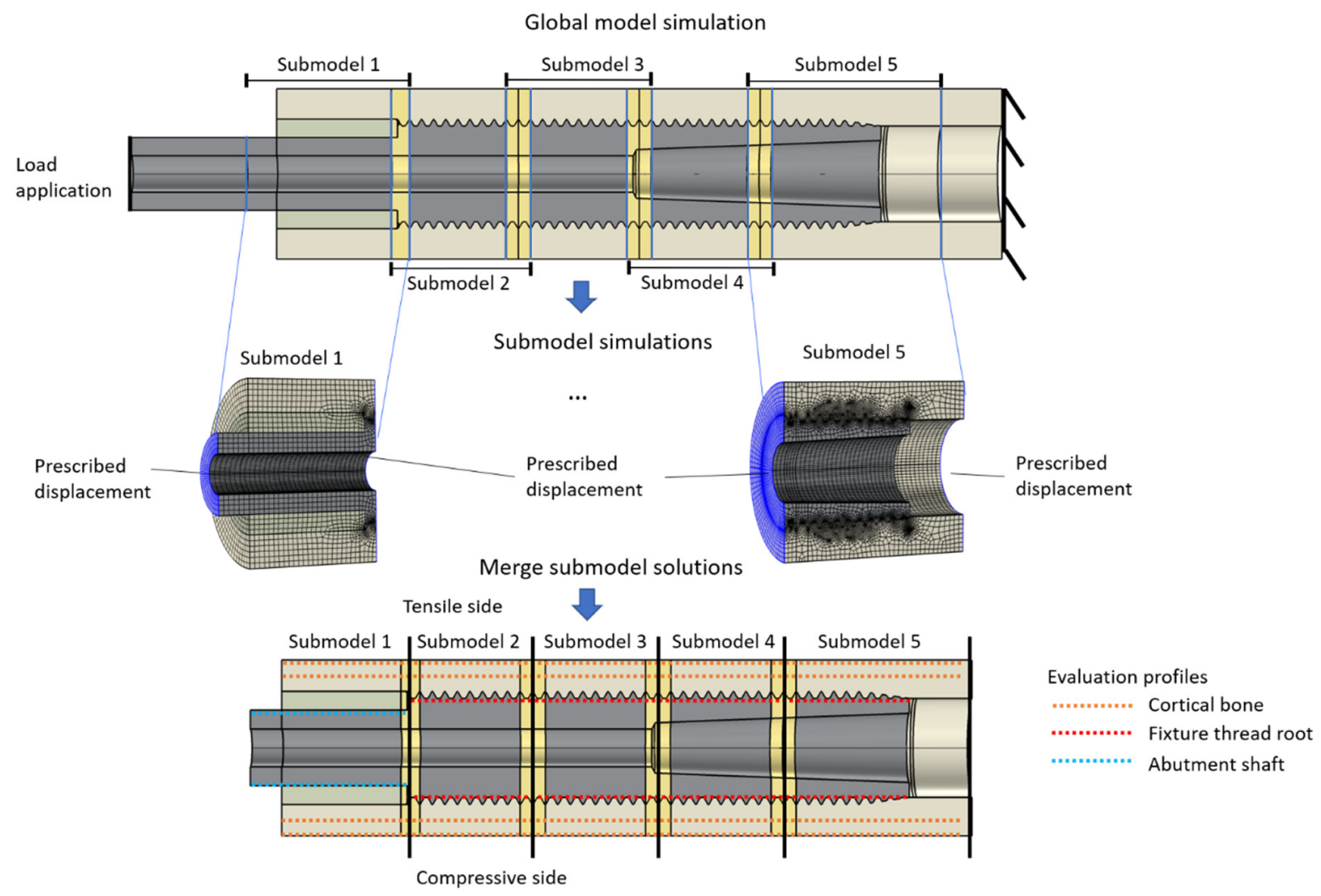

Figure 2. Simulation scheme using submodelling. Top. Global model with submodel partitions. Yellow segments indicate overlapping regions which were included in both of the neighbouring submodels. Middle. Densely meshed submodels. Blue surfaces show where prescribed displacement was assigned from corresponding surfaces in the global model (indicated with blue lines in top image). Bottom. Merging results from each submodel simulation and plotting results along the longitudinal direction at the indicated evaluation profiles.

The material models and material properties used in the simulations are presented in Table 1. Transverse isotropy was assumed for the cortical bone, with uniform material properties around the longitudinal axis. Young's and shear moduli were obtained from the investigation by Reilly and Burstein [42] where the transversely isotropic material properties had been determined based on experimental measurements on human cadaver femora. Single case simulations with the longitudinal Young's modulus of the cortical bone reduced to $12 \mathrm{GPa}$ were also performed. This reduced stiffness may arise due to increased porosity of the cortical bone tissue, which is natural consequence of aging, osteoporosis, and not loading the skeleton in a physiological way, an example being individuals with a lower limb amputation fitted with a socket prosthesis [43]-[45]. The reduction of the longitudinal Young's modulus from $17 \mathrm{GPa}$ to $12 \mathrm{GPa}$ was determined based on experimental data from a study by Dong and Guo [46] where the aforementioned values corresponded to cortical porosity of $8.5 \%$ and $19 \%$ respectively. An additional case of reduced bone support was simulated corresponding to an extreme case of distal resorption, modelled by removing the transplanted bone and the $40 \mathrm{~mm}$ most distal portion of the cortical bone. Two versions of transplanted bone were evaluated, immature transplanted bone with a low Young's modulus representing the state during early rehabilitation, and mature transplanted bone with a higher Young's modulus representing the steady state after full healing and rehabilitation has taken place. Prior histological observation of a tissue biopsy of the transplanted bone retrieved from a revision surgery of an OPRA implant system showed that the mature transplanted bone obtained a trabecular bone structure with a compact bone layer towards 
the soft tissue and the abutment [39]. For simplicity, the transplanted bone was in this study modelled as a homogeneous isotropic tissue with the same material properties as had been used by Stenlund et al. [39]. The fixture and the abutment are manufactured from Ti6Al4V and were also modelled with isotropic material properties.

Table 1. Material models and material properties used in the FE-models

\begin{tabular}{lll} 
Component & Material model & Material properties \\
\hline Fixture and abutment & Isotropic & $\mathrm{E}=110 \mathrm{GPa}$ \\
& & $v=0.3$ \\
\hline Normal cortical bone & Transversely isotropic & $\mathrm{E}_{\mathrm{r}}=\mathrm{E}_{\Theta}=11.5 \mathrm{GPa}, \mathrm{E}_{\mathrm{z}}=17 \mathrm{GPa}$ \\
& & $\mathrm{G}_{\mathrm{r} \theta}=3.6 \mathrm{GPa}, \mathrm{G}_{\mathrm{rz}}=\mathrm{G}_{\Theta z} 3.3 \mathrm{GPa}$ \\
& $v_{\mathrm{r} \theta}=v_{\mathrm{rz}}=v_{\Theta z}=0.3$ \\
& & $\mathrm{E}_{\mathrm{r}}=\mathrm{E}_{\Theta}=11.5 \mathrm{GPa}, \mathrm{E}_{\mathrm{z}}=12 \mathrm{GPa}$ \\
& $\mathrm{G}_{\mathrm{r} \theta}=3.6 \mathrm{GPa}, \mathrm{G}_{\mathrm{rz}}=\mathrm{G}_{\Theta z} 3.3 \mathrm{GPa}$ \\
Osteoporotic cortical & Transversely isotropic & $v_{\mathrm{r} \theta}=v_{\mathrm{rz}}=v_{\Theta z}=0.3$ \\
bone & & $\mathrm{E}=4 \mathrm{GPa}$ \\
& & $v=0.3$ \\
\hline Mature transplanted & Isotropic & $\mathrm{E}=0.4 \mathrm{GPa}$ \\
bone & & $v=0.3$ \\
\hline Immature & Isotropic & \\
transplanted bone & &
\end{tabular}

From studies obtaining direct load data at distal end of the abutment, it is known that at physiological loading during activities of daily living, the bending moment is the main contributor to the stress state in the implant and the bone, and the longitudinal force is the dominating force component [22]. The studied load cases were therefore reduced to only include only these components. This led to symmetric loading which in combination with the geometric symmetry allowed for the model reduction as described above. The first load case, LC1, refers to high loading ambulation. The highest body weight acceptable for treatment with the OPRA implant system is $100 \mathrm{~kg}$. During walking this corresponds to a maximum longitudinal force of approximately $1000 \mathrm{~N}$. This value was therefore chosen for the longitudinal force component for LC1. The bending moment was derived from a study conducting load measurements at the distal end of the abutment in 20 individuals with the OPRA implant system at the transfemoral level while performing everyday ambulatory activities [22]. The highest measured bending moment reported during any of the investigated activities within that study was $90 \mathrm{Nm}$ (observed in a single participant during stairs descent). This value was therefore chosen as the value for the bending moment in LC1. The second load case, LC2, refers to average loading during level ground walking. The magnitudes of the longitudinal force and the bending moment were also derived from the aforementioned study. They correspond to the mean peak longitudinal force and mean peak bending moment measured across the study participants during level ground walking.

Table 2. Investigated load cases.

\begin{tabular}{ccll} 
Load case & F & M & Interpretation \\
\hline LC1 & 1000 & 90 & High ambulatory loading \\
LC2 & 625 & 37 & Average ambulatory loading
\end{tabular}

The FE meshes of both the global model and the submodels were created by first creating a structured surface mesh on a longitudinal section of the axisymmetric FE-geometry. The surface mesh was refined in the threaded portion of the geometry. The surface mesh was then swept around the longitudinal axis to form a 3D mesh. This meshing strategy was chosen to minimize the number of 
elements in the model while ensuring a high mesh resolution in the threaded region. The resulting mesh consisted of second order hexahedron and prismatic elements. The total number of elements were 90 000-120 000 for the global models and 35 000-80 000 for each sub-model depending on variations in geometry (i.e., variations in $t, r_{\text {root }}, d$, and $p$ ). A mesh convergence analysis along a longitudinal profile along the fixture thread root, the region most influenced by the quality of the mesh, confirmed that further mesh refinement from the chosen mesh parameters led to $<3 \%$ change in results anywhere along the profile.

\section{Results}

LC1 and LC2 both correspond to repeated loading conditions for which tensile stresses in general are considered more harmful than compressive stresses [47]. For this reason, only the stress and strain state on the tensile side of the implant-bone model is reported in this study.

\section{Analytical estimation of load sharing}

The result from the analysis of the simplified geometry, considering the implant and the cortical bone as two uniform cylinders is shown in Figure 3 along with the estimated changes in the peak stress in the fixture for varying thread depth and cortical thickness.

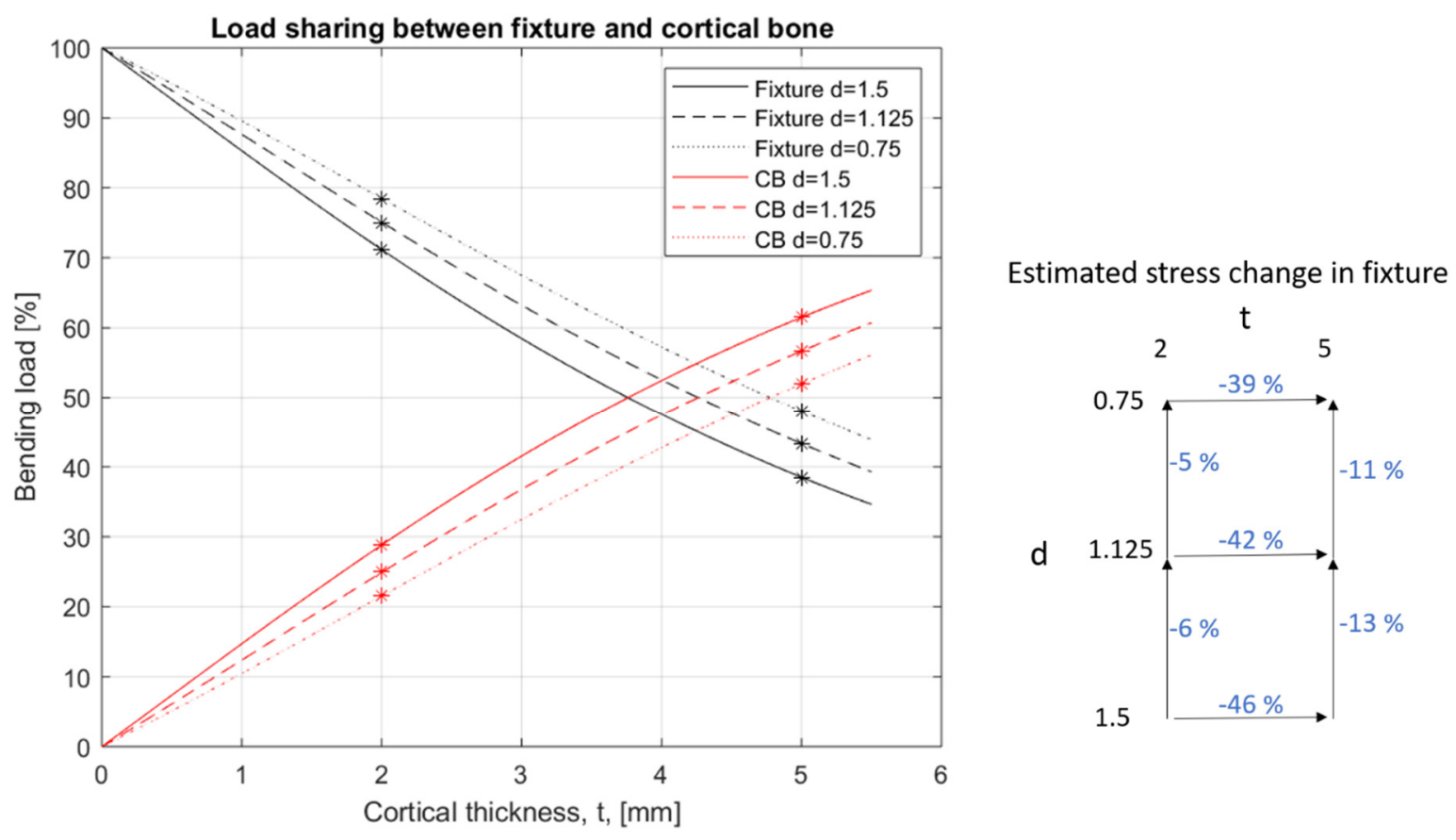

Figure 3. Estimated load sharing between fixture and cortical bone (CB) from analysis of simplified geometry. To the right, the estimated change in peak stress in the fixture for varying thread depth and cortical thickness.

\section{Stress in fixture}

Thread roots constitute stress concentrations with locally elevated stress levels [48]. The thread root of the fixture may therefore be susceptible to high stress levels and could be a potential location of failure if exposed to excessive loading. The longitudinal stress in the thread root of the tensile side of the fixture subjected to LC1 is presented in Figure 4 for three different thread depths and for two different cortical thicknesses. 


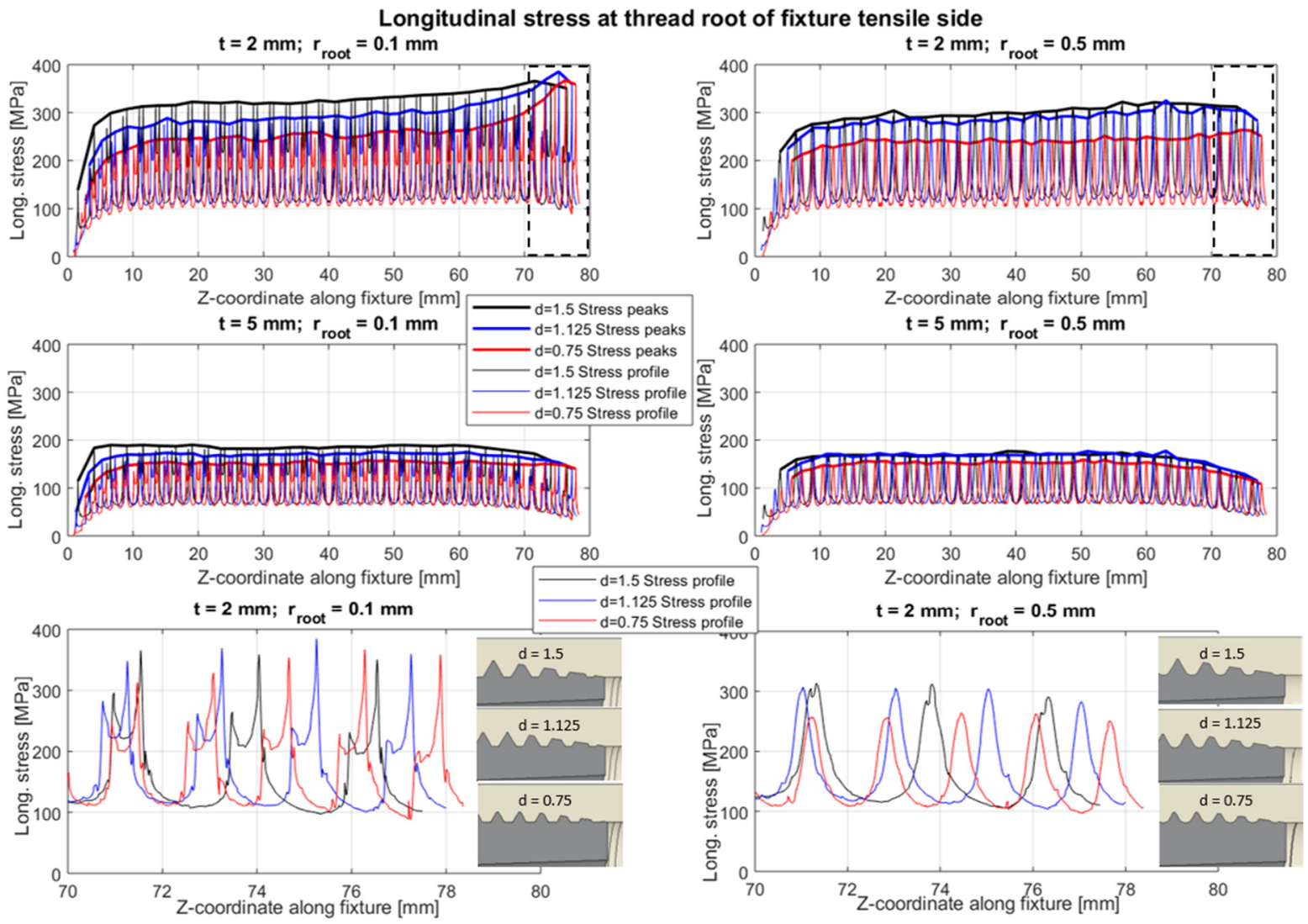

Figure 4. Longitudinal stress in tensile side of the fixture thread root during LC1. Top. Results from simulations of three different values of the thread depth, $d$. Cortical thickness, $t$, and fixture thread root radius, $r_{\text {root }}$ are kept constant at $2 \mathrm{~mm}$ and $0.5 \mathrm{~mm}$ respectively. Middle. Results from simulations of three different values of the thread depth, $d$. Cortical thickness, $t$, and fixture thread root radius, $r_{\text {root }}$ are kept constant at $5 \mathrm{~mm}$ and $0.5 \mathrm{~mm}$, respectively. Bottom. Results from the proximal end of the fixture with cortical thickness $t=2 \mathrm{~mm}$ (zoomed in from the dashed regions within the top graphs). The proximal geometry of the fixture is also shown.

At the distal end of the fixture, $(z=0)$, the peak stresses are reduced in all studied cases. This is also observed at the proximal end of the fixture for the cases with the large cortical thickness, $(t=5 \mathrm{~mm})$. However, for the case with small cortical thickness $(t=2 \mathrm{~mm})$, there is an increase in the peak stress in the fixture towards the proximal end especially for the two more shallow thread depths with a small thread root radius. Between the z-coordinates, $20 \mathrm{~mm}$ and $60 \mathrm{~mm}$ along the fixture, the differences between the peak stress profiles are approximately constant, thus the effect of each parameter can be approximated by an average over this region without losing much detail. Such summarized presentation of the parameter evaluation is shown in Figure 5. 


\section{Longitudinal stress peaks at fixture thread root tensile side}

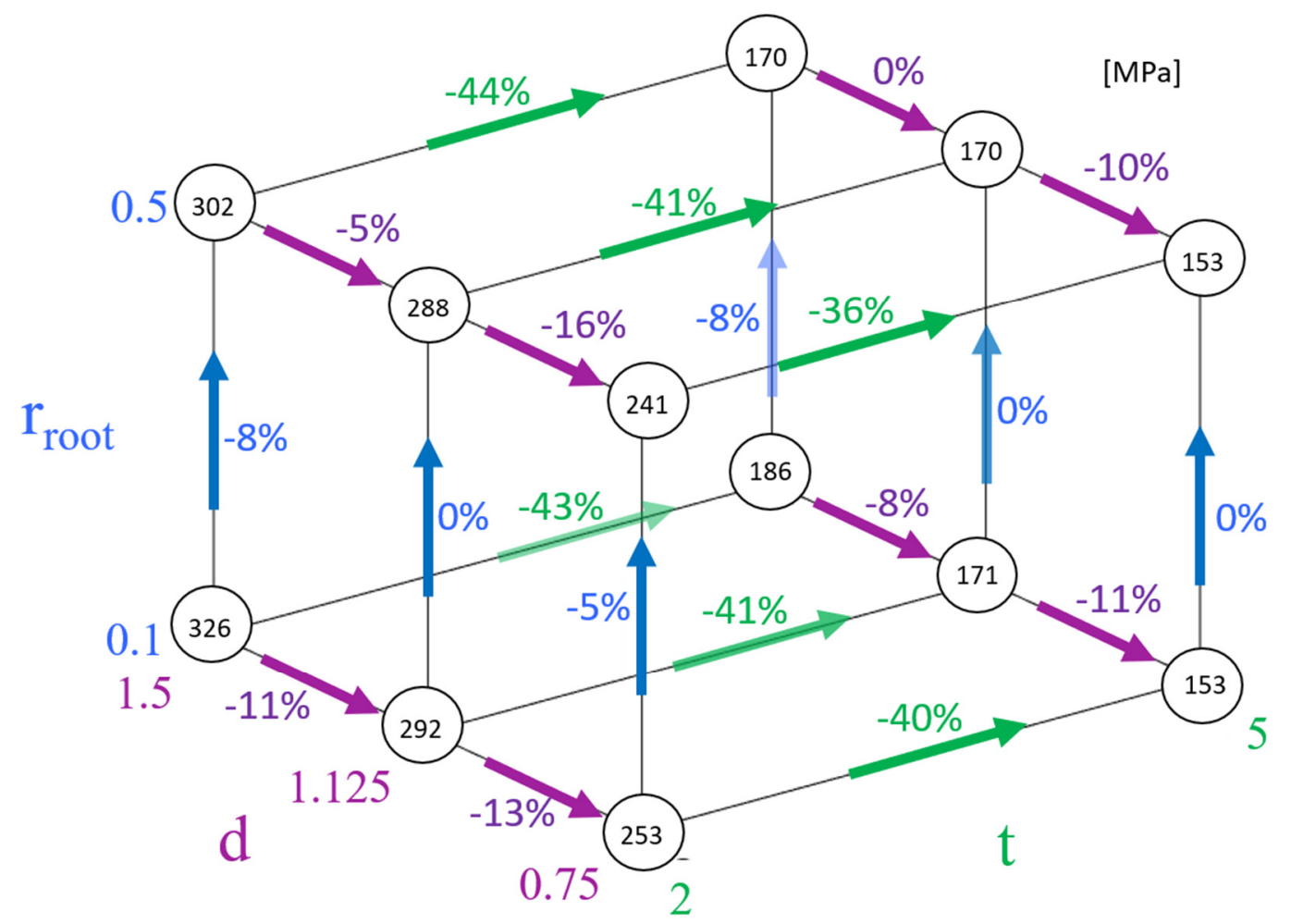

Figure 5. Parameter evaluation of the longitudinal stress in the thread root of the fixture during LC1. Circled values denote the average peak longitudinal stress (MPa) in the fixture thread root between z-coordinates $20 \mathrm{~mm}$ and $60 \mathrm{~mm}$ during LC1. Colored values denote the percentage difference in the results when varying a parameter.

Within the investigated parameter space, the difference between $2 \mathrm{~mm}$ and $5 \mathrm{~mm}$ cortical thickness had the largest effect on the peak stresses in the fixture thread root, with approximately $40 \%$ reduction across different thread depths and root radii. A reduced thread depth from $1.5 \mathrm{~mm}$ to 0.75 $\mathrm{mm}$ led to $20 \%-22 \%$ reduction with $\mathrm{t}=2 \mathrm{~mm}$ and $10 \%-18 \%$ reduction with $\mathrm{t}=5 \mathrm{~mm}$. The effect of an increased radius in the thread root from $0.1 \mathrm{~mm}$ to $0.5 \mathrm{~mm}$ had a less prominent effect, and stress reductions were only noted for the most deep and shallow threads at $t=2 \mathrm{~mm}$ (Figure 6) and the deepest thread at $\mathrm{t}=5 \mathrm{~mm}$. In the three other simulated cases, no change in the average peak stress was noted. 


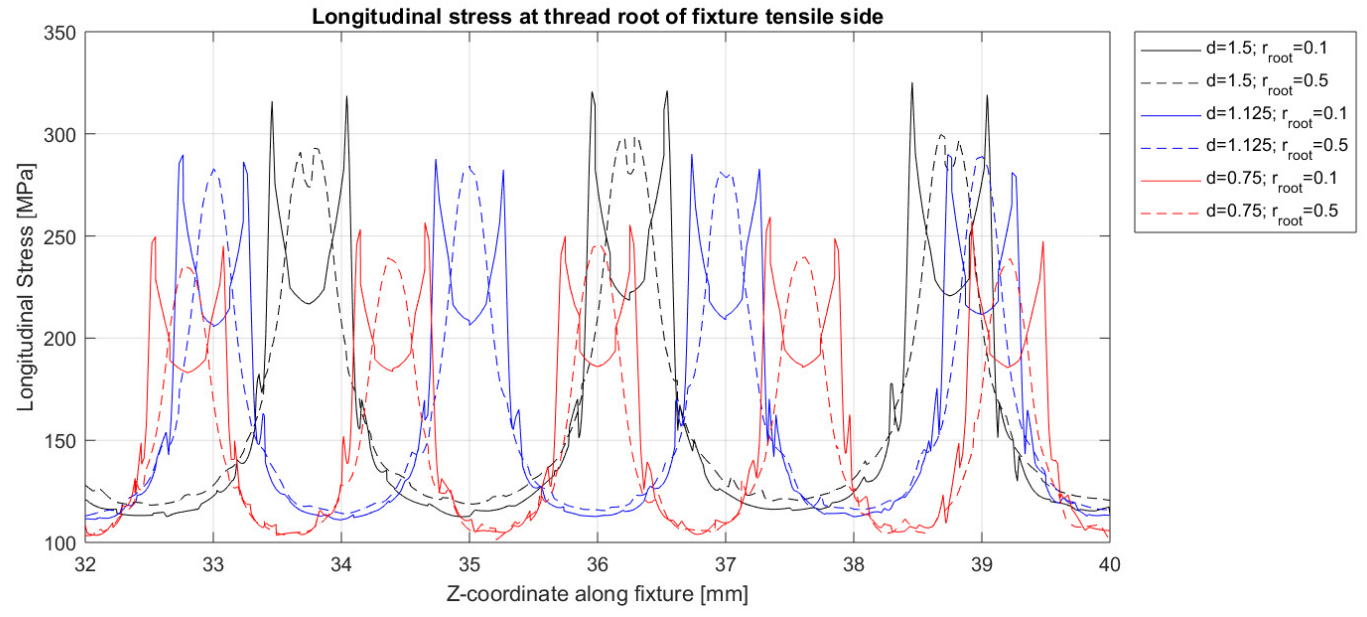

Figure 6. Longitudinal stress in tensile side of the fixture thread root during LC1 for different thread depths and thread root radii

The separate effects of reduced cortical stiffness and extreme distal bone resorption on the maximum stress in the thread root of the fixture are shown in Figure 7. On average, a reduction in the longitudinal cortical stiffness from $17 \mathrm{GPa}$ to $12 \mathrm{GPa}$ resulted in $12 \%$ and $30 \%$ increases in maximum longitudinal stress for $2 \mathrm{~mm}$ and $5 \mathrm{~mm}$ cortical thickness, respectively. In the case of extreme bone resorption, the maximum stress increased by $54 \%$ on average compared with a situation of full uniform cortical bone support $(\mathrm{t}=2 \mathrm{~mm})$. 


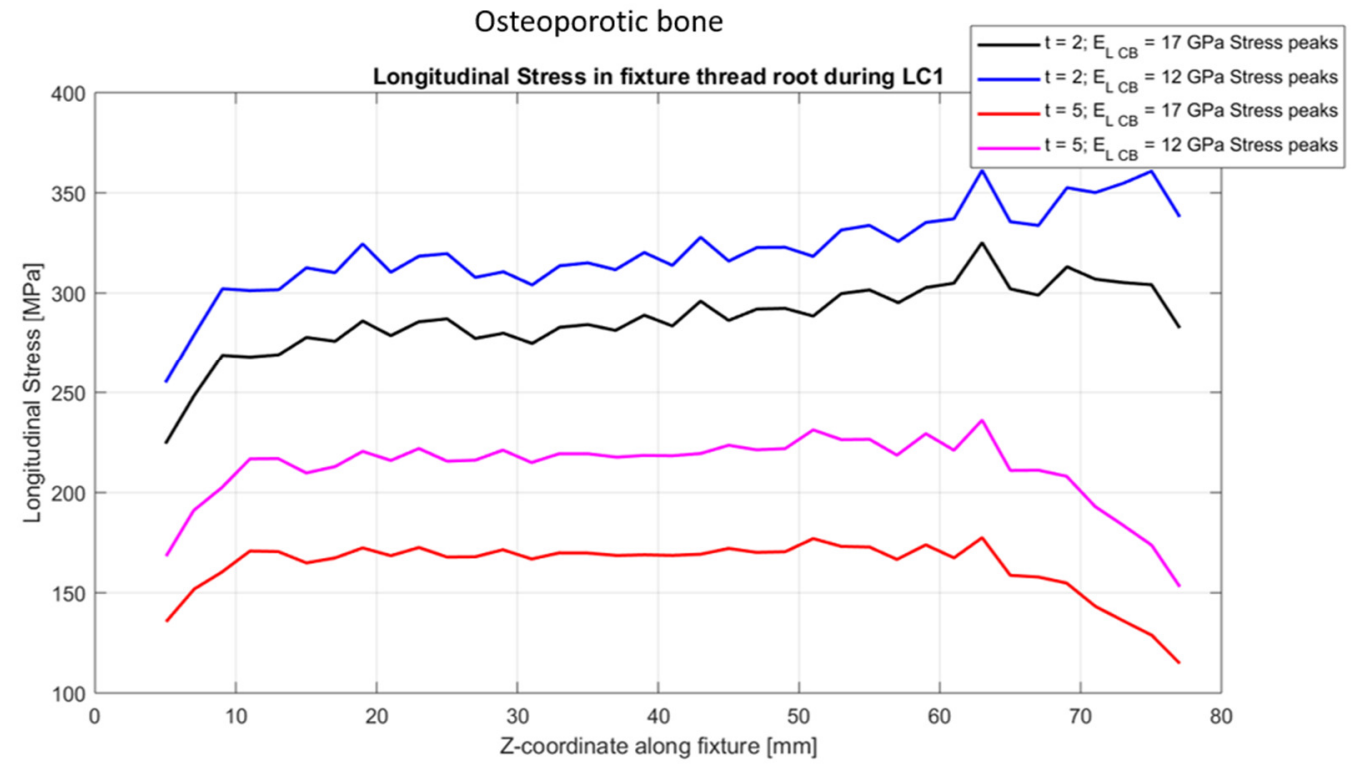

Extreme distal bone resorption
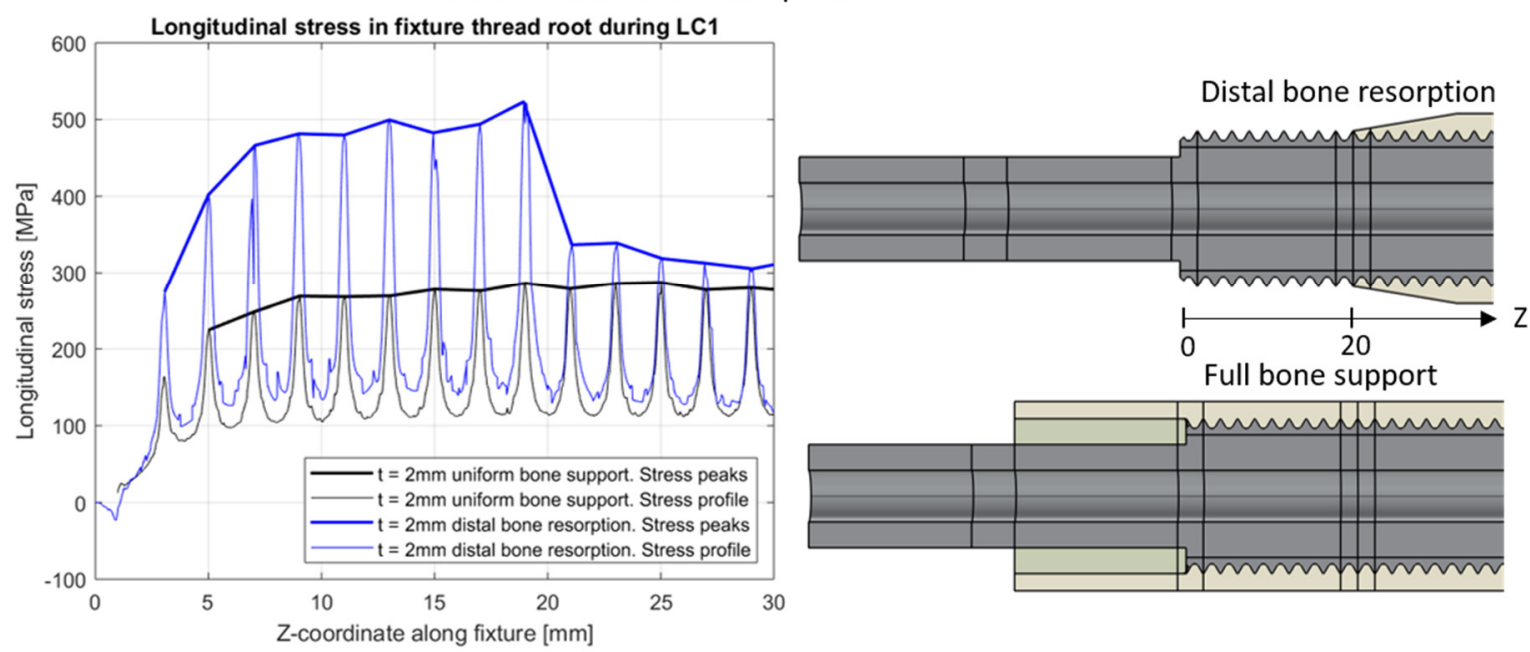

Figure 7. Top. The longitudinal stress peaks in the fixture thread root for two different values of longitudinal stiffness in the cortical bone and for two different cortical thicknesses during LC1. Bottom. The effect of extreme distal bone resorption during $L C 1$.

\section{Stress in abutment}

A comparison of the longitudinal stress on the tensile side of the abutment shaft for different configurations of cortical thickness and Young's modulus for the transplanted bone is presented in Figure 8. With respect to the case without any bone support (extreme distal bone resorption) a maximum stress reduction of $31 \%$ was achieved with $5 \mathrm{~mm}$ cortical thickness and a Young's modulus of $4 \mathrm{GPa}$ in the transplanted bone. Interestingly, even the smallest cortical thickness $(t=2 \mathrm{~mm})$ and a very soft transplanted bone $\left(\mathrm{E}_{\mathrm{TB}}=0.4 \mathrm{GPa}\right)$ resulted in up to $10 \%$ reduction in the stress of the abutment. 


\section{Longitudinal stress in tensile side of abutment}
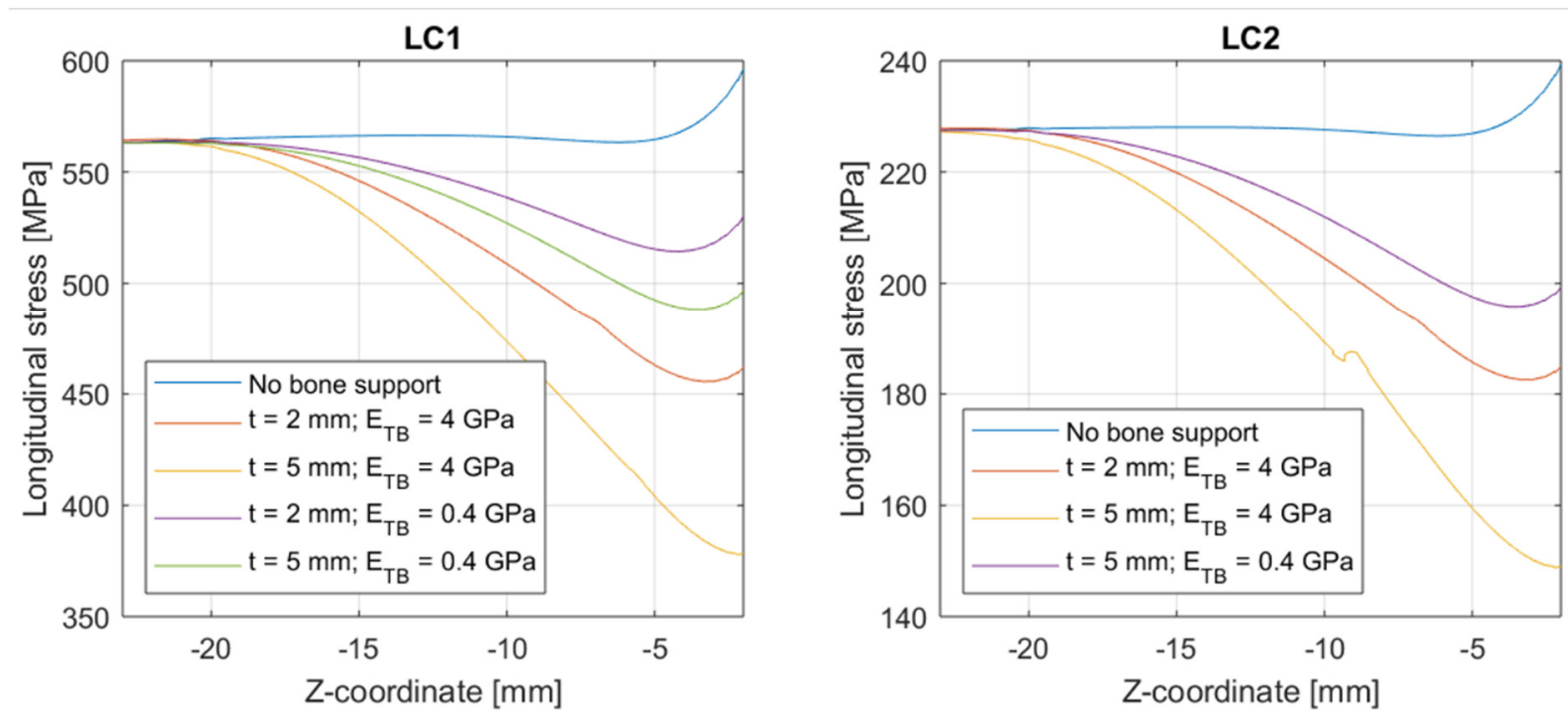

Figure 8. Longitudinal stress on the tensile side of the abutment shaft during LC1 and LC2.

\section{Stress and strain in cortical bone}

The longitudinal stress and strain along the tensile side of the cortical bone during is shown in Figure 9. To show the effect of the cortical thickness and the cortical stiffness, all graphs presented in Figure 10 are from simulations with fixed thread depth $d=1.125 \mathrm{~mm}$ and fixed thread root radius $r_{\text {root }}=0.5$ $\mathrm{mm}$. There were clear maxima in the longitudinal stress and longitudinal strain at the z-coordinates corresponding to each end of the fixture. Especially large were the maxima at the proximal end for the simulated cases with $2 \mathrm{~mm}$ cortical thickness. At the outer cortex at this location the maximum longitudinal stress reached $109 \mathrm{MPa}$ and $43 \mathrm{MPa}$ for LC1 and LC2 respectively for the simulations with ordinary cortical stiffness $\left(E_{L C B}=17 \mathrm{GPa}\right.$ ). For the $5 \mathrm{~mm}$ cortical thickness cases the maximum stress was $43 \mathrm{MPa}$ at the outer cortex. At the distal end of the fixture the maximum stress in the cortical bone reached at most $42 \mathrm{MPa}$ and $27 \mathrm{MPa}$ for the simulations with $2 \mathrm{~mm}$ and $5 \mathrm{~mm}$ cortical thickness, respectively.

As a natural consequence of Hooke's law, a reduction of the cortical stiffness led to reduced stress but increased strains. The strains at the proximal end of the fixture were the largest, locally reaching $7940 \mu \varepsilon$ for the softer cortical bone simulation and $6050 \mu \varepsilon$ for the normal cortical bone simulation with $2 \mathrm{~mm}$ bone thickness in LC1. In LC2 the peak strain reached $2370 \mu \varepsilon$ with ordinary cortical bone stiffness.

The largest strains were observed for the case with the reduced cortical stiffens ( $E_{L C B}=12 \mathrm{GPa}$ ) reaching a maximum of $7940 \mu \varepsilon$ locally for LC1. At ordinary cortical stiffness, the corresponding maximum strain was $6050 \mu \varepsilon$ and $2370 \mu \varepsilon$ respectively for LC1 and LC2. 
Cortical bone LC1
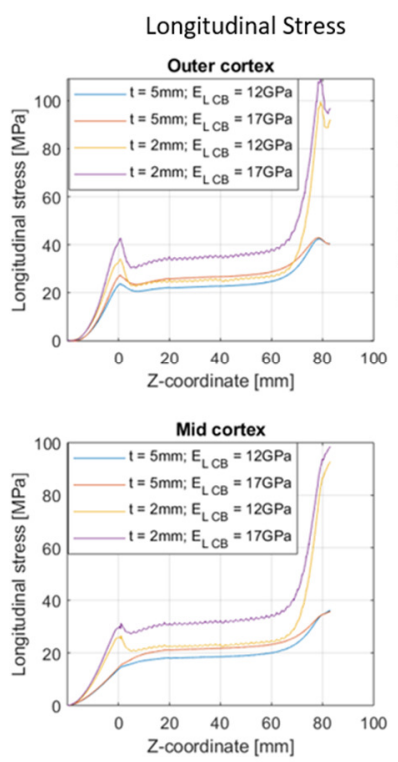
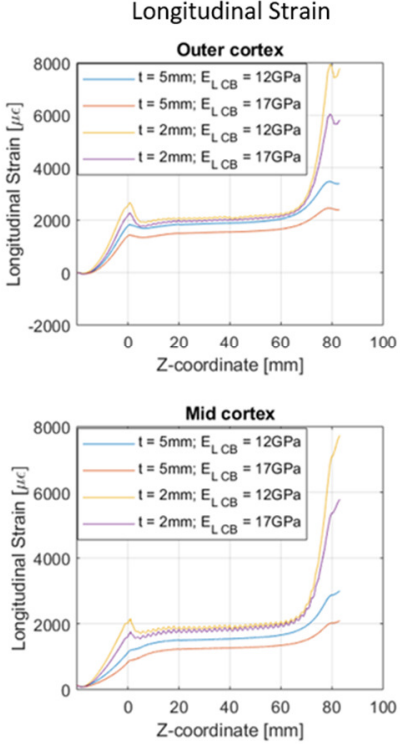

Cortical bone LC2
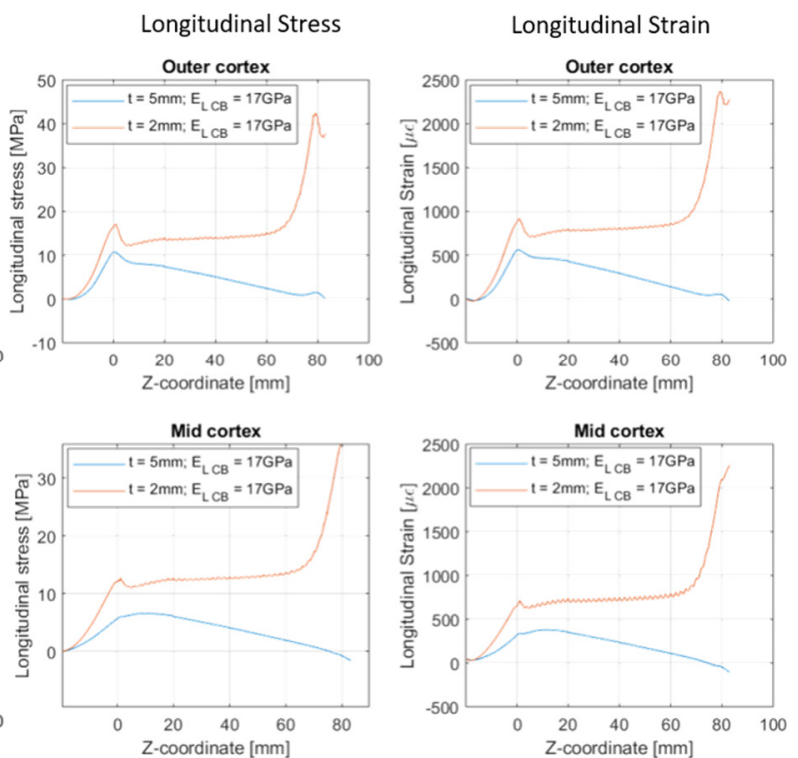

Figure 9. Longitudinal stress and strain in the cortical bone during LC1 and LC2. For the results presented in the graphs the parameters $d$, and $r_{\text {root, }}$ were kept fixed at $1.125 \mathrm{~mm}$ and $0.5 \mathrm{~mm}$ respectively.

In Figure 10, the effect of the thread profile parameters, $d$ and $r_{\text {root }}$ on the longitudinal stress and longitudinal strain in the cortical bone is shown. The presented graphs are both from LC1 and at cortical thickness $t=2 \mathrm{~mm}$. At the outer cortex along the length of the fixture, higher stress and strains are observed for the simulations with large thread depth, except for at locations corresponding to the ends of the fixture. At the z-coordinate corresponding to the proximal end of the fixture longitudinal stress and longitudinal strain is lower in simulations with large thread depth. The highest values for the longitudinal stress and longitudinal strain in the cortical bone was observed at the proximal end of the fixture for the shallowest thread depth and the smallest fixture thread root radius and smallest cortical thickness.

Cortical thickness $\mathrm{t}=2 \mathrm{~mm}$

Longitudinal stress and strain at outer cortex during LC1
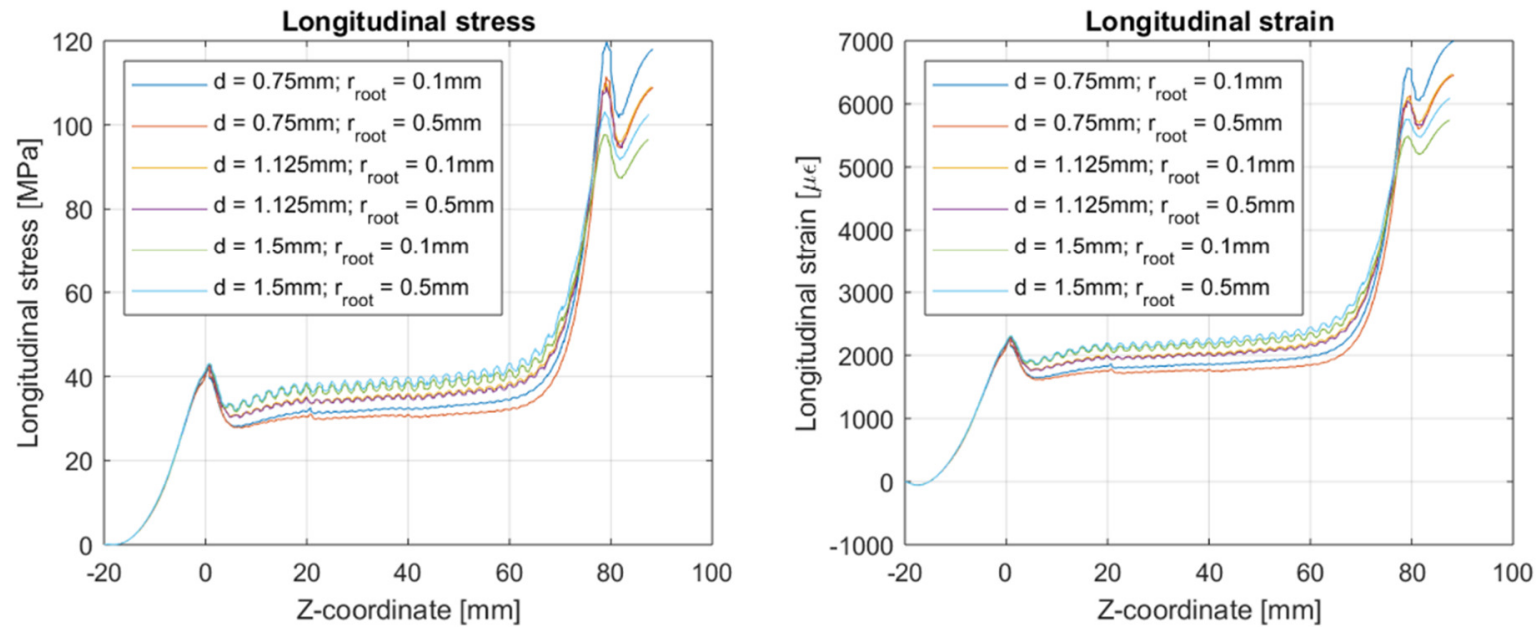

Figure 10. Longitudinal stress and strain during LC1. The parametert is kept constant at $2 \mathrm{~mm}$ in the graphs. 


\section{Discussion}

We conducted an FE-based evaluation of a generic model of a bone-anchored implant system inspired by the OPRA implant system that was subjected to external loads corresponding to average and high ambulatory loading. Within this investigation, a parametric analysis has been carried out to determine how the peak stress and strains in implant system and the periprosthetic bone are affected by the cortical thickness, the thread depth and the thread root radius. Single case investigations have been performed to quantify the effects of reduced cortical stiffness and distal bone resorption.

\section{Stress in fixture}

The initial analytical approximation of the cortical bone and the fixture as two cylindrical components without thread provided preliminary predictions for the results from the FE-simulations. Changes in the cortical thickness and thread depth, each lead to changes in the external diameter of respective component. Changes in the cortical thickness had the largest effect on the longitudinal stresses in the thread root of the fixture. This was expected considering the external diameter's high influence on the area moment of inertia (Eq. 1). The average reductions of the peak longitudinal stress in the thread root of the fixture, when increasing the cortical thickness from $2 \mathrm{~mm}$ to $5 \mathrm{~mm}$, were similar between the FE-simulations ( $36 \%-44 \%$ ) and the predicted values (39\% - $46 \%$ ).

The effect of the thread depth was influenced by the cortical thickness. With cortical thickness fixed at $\mathrm{t}=2 \mathrm{~mm}$, reducing the thread depth from $1.5 \mathrm{~mm}$ to $0.75 \mathrm{~mm}$, led to $20 \%$ and $22 \%$ reduction in the average peak stress in the thread root of the fixture with a large and small root radius respectively. With cortical thickness fixed at $\mathrm{t}=5 \mathrm{~mm}$, the effect of an identical reduction in thread depth $(1.5 \mathrm{~mm}$ to $0.75 \mathrm{~mm}$ ), led to smaller reductions ( $10 \%$ and $18 \%$ for large and small root radius respectively). The reason for the smaller influence of the thread depth at the larger cortical thickness can be attributed to the same reason as described above, namely the external diameter's importance for the bending stiffness (Eq. 1).

Interestingly, for a cortical thickness of $2 \mathrm{~mm}$ and a small root radius, the magnitude of the stress peaks increased towards the proximal end of the fixture for the two shallower threads (Figure 4). At the proximal end of the fixture the internal diameter of the fixture was $11 \mathrm{~mm}$ for all models whereas the external diameter of the fixture was dependent on the thread depth (Figure 1). As a result, the external diameter and material thickness of the fixture was larger for models with smaller thread depth. This led to that the proximal stiffness of the fixture was larger in models with small thread depth and that the extent of proximal stiffness reduction of the fixture (due to the chamfered external diameter proximally) was smaller in these FE-models. This may offer one explanation for the observed increased peak stress at the thread root of the proximal fixture for the shallower threads at $2 \mathrm{~mm}$ cortical thickness in Figure 4.

The effect of reduced cortical stiffness was larger for the situation with large cortical thickness, since in this case the cortical bone is resisting a larger portion of the total load and thus a reduction in stiffness consequently has a larger effect on the stresses in the Fixture (Eq. 1). The simulation with extreme distal bone resorption led to a $54 \%$ increase in the peak stresses in the fixture thread root compared with the reference case with uniform bone support with $2 \mathrm{~mm}$ cortical thickness. Such increase in the stresses might challenge the mechanical integrity of the fixture and thus, the resorption should be addressed before reaching such extremes.

\section{Stress in abutment}

The stress in the abutment was higher than the stress in the fixture, an effect of the smaller external diameter and the conscious design decision to make the abutment the weaker component, since it 
can be more easily replaced if damaged than the fully implanted and osseointegrated fixture. The simulations showed that the transplanted bone can contribute to substantial stress reductions in the abutment, especially proximally corresponding to the region where the abutment exits from the fixture and would otherwise be particularly vulnerable. The transplanted bone bridges the gap between the distal cortical bone and the abutment and thus the cortical bone may contribute to resisting the bending loads. The advantage of the transplanted bone was observed with material properties representing immature transplanted bone, and improved as the stiffness increased.

\section{Cortical bone}

With minimum cortical thickness $(2 \mathrm{~mm})$, the maximum longitudinal stress reached above $100 \mathrm{MPa}$ at the outer cortex at the level of the proximal end of the fixture during LC1. For the $5 \mathrm{~mm}$ cortical thickness cases, the maximum stress was $43 \mathrm{MPa}$. At the distal end of the fixture the peak stress in the cortical bone reached at most $42 \mathrm{MPa}$ for the case with $2 \mathrm{~mm}$ cortical thickness. For LC2 loading, the maximum stress reached $42 \mathrm{MPa}$ at the level of the proximal end of the fixture. A reduction of the cortical stiffness led to reduced stress but increased strains. The strains at the proximal end of the fixture were the largest, locally reaching up to $8000 \mu \varepsilon$ for the softer cortical bone simulation and around $6000 \mu \varepsilon$ for the normal cortical bone simulation with $2 \mathrm{~mm}$ bone thickness in LC1 (Figure 4). These strain levels are in the order of the yield strain reported from previous research [49]. In the FEsimulations, a small thread depth led to higher peak longitudinal stress and longitudinal strain in the cortical bone at to the proximal end of the fixture compared with a larger thread depth (Figure 10). This may be explained by the fact that a smaller thread depth led to reduced proximal cortical bone thickness due to its geometrical definition in the FE-models as $t+d$ (Figure 1). Along the fixture length between the local maxima at each end of the fixture, higher longitudinal stress and longitudinal strains were instead observed for the simulations with large thread depth. This may be explained by the fact that the fixtures with larger thread depth provide less stress shielding of the bone due to their smaller core diameter, thereby leading to increased strains and stresses in the cortical bone.

For LC2 the peak strain was $2370 \mu \varepsilon$ for cortical bone with ordinary stiffness and $2 \mathrm{~mm}$ cortical thickness. For the case with $5 \mathrm{~mm}$ cortical thickness there were only minor local stress and strain peaks at the proximal end of the fixture location. It has been suggested that peak longitudinal strains above $1000 \mu \varepsilon$ leads to endosteal and periosteal bone formation but little change intracortically, whereas peak longitudinal strains below $1000 \mu \varepsilon$ leads to bone loss due to endosteal resorption and increased intracortical porosity [50]. This would suggest that during both LC1 and LC2 new bone formation at the proximal end of the fixture is to be expected, at least locally in the plane which is subjected to the highest bending moment. This would lead to an increased cortical thickness with subsequent, local decrease in stress and strain and a reduced risk of periprosthetic fracture in case of overloading.

\section{Limitations}

There are discrepancies between the geometry of FE-model and the clinically used implant system. As mentioned above, the proximal stiffness reduction of the fixture was not uniform between models of different thread depths. This led to that especially the FE-models with shallow thread depths had unrealistically high stiffness proximally. Furthermore, the FE-models lacked longitudinal slots in the proximal fixture, a feature present in the clinically used fixtures. Slots act to reduce proximal stiffness and therefore, it can be assumed that the stress and strain concentrations in the cortical bone at the proximal end of the fixture are more severe in the FE-model compared with the clinically used implant. A detailed investigation of the effect of the proximal fixture stiffness on the stress and strain in the cortical bone at the proximal end of the fixture was outside the scope of this evaluation and would require a separate study. Another difference between the FE-geometry and the clinically used implant 
system is that the fixture and abutment were modelled as a single component rather than as separate components, thus omitting any effects from the contacting interface. Furthermore, the abutment screw and the preload were disregarded in the FE-model. This led to the omission of the resulting compressive stresses in the abutment shaft and thus, the presented longitudinal stress on the tensile side of the abutment shaft may be exaggerated by approximately $50 \mathrm{MPa}$ compared with the clinical scenario. The geometry of the bone was modelled as a cylinder with uniform external diameter with the implant concentrically placed within the bone. This is a simplification from individual clinical cases which may vary both in the bone geometry and the implant placement. The load cases used in this study were derived from in vivo experimentally measured longitudinal forces and bending moments, but no torsional load components were considered. Furthermore, the variability in loading between users is known to be large [22], thus the magnitudes of the forces and moments may not be applicable to describe any individual user.

\section{Conclusion}

We conducted a numerical simulation (FE) of a bone-anchoring implant system. We found that the cortical thickness had the largest effect on stress and strain in the cortical bone and the fixture. At loads corresponding to high loading ambulation, $5 \mathrm{~mm}$ of cortical bone thickness led to approximately $40 \%$ lower maximum longitudinal stresses at the central half of the fixture compared with a cortical thickness of $2 \mathrm{~mm}$. Changes within the investigated range of thread profile parameters had a smaller effect $(0 \%-8 \%$ stress reduction for a thread root radius of $0.5 \mathrm{~mm}$ as compared with $0.1 \mathrm{~mm}$, and $10 \%-22 \%$ stress reduction for thread depth of $0.75 \mathrm{~mm}$ as compared to $1.5 \mathrm{~mm}$ ). At the location where the abutment exits from the fixture, autologously transplanted bone may provide substantial support for the abutment by activating support from surrounding cortical bone. We observed $10 \%$ and $31 \%$ stress reduction in the abutment for simulations with transplanted bone with Young's modulus of $0.4 \mathrm{GPa}$ and $4 \mathrm{G} \mathrm{Pa}$, respectively. Reduced cortical bone stiffness led to increased stresses in the fixture. A $29 \%$ reduction in the longitudinal Young's modulus of the cortical bone led to a $12 \%$ and $30 \%$ increase in maximum stress in the fixture at cortical thickness $2 \mathrm{~mm}$ and $5 \mathrm{~mm}$, respectively. If distal bone resorption would occur to the extent that a portion of the distal fixture is without support from cortical bone, the maximum stress in the fixture may increase by up to $54 \%$ as compared with full cortical support of $2 \mathrm{~mm}$ cortical thickness.

The results from this investigation provides quantitative information on the interplay between geometrical and material parameters of the implant system and surrounding bone, and their effects on the stress and strain in respective material. This may inform further development of implant systems for bone-anchored attachment of limb prostheses.

\section{References}

[1] H. E. J. Meulenbelt, J. H. B. Geertzen, M. F. Jonkman, and P. U. Dijkstraa, "Skin problems of the stump in lower limb amputees: 2 . influence on functioning in daily life," Acta Derm. Venereol., 2011.

[2] F. T. Hoaglund, H. E. Jergesen, L. Wilson, L. W. Lamoreux, and R. Roberts, "Evaluation of problems and needs of veteran lower-limb amputees in the San Francisco Bay Area during the period 1977-1980.," J. Rehabil. R\&D, 1983. 
[3] N. L. Dudek, M. B. Marks, S. C. Marshall, and J. P. Chardon, "Dermatologic conditions associated with use of a lower-extremity prosthesis," Arch. Phys. Med. Rehabil., vol. 86, no. 4, pp. 659-663, 2005.

[4] K. Hagberg and R. Brånemark, "Consequences of non-vascular trans-femoral amputation: a survey of quality of life, prosthetic use and problems.," Prosthet. Orthot. Int., vol. 25, no. 3, pp. 186-194, 2001.

[5] M. W. Legro et al., "Issues of importance reported by persons with lower limb amputations and prostheses.," J. Rehabil. Res. Dev., vol. 36, no. 3, pp. 155-63, 1999.

[6] R. Sherman, "Utilization of prostheses among US veterans with traumatic amputation: a pilot survey," J. Rehabil. Res. Dev., vol. 36, no. 2, pp. 100-8, 1999.

[7] R. Brånemark, Ö. Berlin, K. Hagberg, P. Bergh, B. Gunterberg, and B. Rydevik, "A novel osseointegrated percutaneous prosthetic system for the treatment of patients with transfemoral amputation: A prospective study of 51 patients," Bone Jt. J., vol. 96 B, no. 1, pp. 106-113, 2014.

[8] P. I. Branemark, "Osseointegration and its experimental background," J. Prosthet. Dent., vol. 50, no. 3, pp. 399-410, 1983.

[9] "Osseointegration," Merriam-Webster Medical Dictionary. [Online]. Available: https://www.merriam-webster.com/medical/osseointegration. [Accessed: 22-Mar-2021].

[10] R. Brånemark, P.-I. Brånemark, B. Rydevik, and R. R. Myers, "Osseointegration in skeletal reconstruction and rehabilitation," J. Rehabil. Res. Dev., vol. 38, pp. 175-181, 2001.

[11] E. Eriksson and P.-I. Brånemark, "Osseointegration from the perspective of the plastic surgeon.," Plast. Reconstr. Surg., vol. 93, no. 3, pp. 626-637, 1994.

[12] K. Hagberg, E. Hansson, and R. Brånemark, "Outcome of percutaneous osseointegrated prostheses for patients with unilateral transfemoral amputation at two-year follow-up," Arch. Phys. Med. Rehabil., vol. 95, no. 11, pp. 2120-2127, 2014.

[13] K. Hagberg, S.-A. Ghassemi Jahani, K. Kulbacka-Ortiz, P. Thomsen, H. Malchau, and C. Reinholdt, "A 15-year follow-up of transfemoral amputees with bone-anchored transcutaneous prostheses," Bone Joint J., 2020.

[14] E. Häggström, K. Hagberg, B. Rydevik, and R. Brånemark, "Vibrotactile evaluation: osseointegrated versus socket-suspended transfemoral prostheses.," J. Rehabil. Res. Dev., vol. 50, no. 10, pp. 1423-34, 2013.

[15] R. Tranberg, R. Zügner, and J. Kärrholm, "Improvements in hip- and pelvic motion for patients with osseointegrated trans-femoral prostheses," Gait Posture, vol. 33, no. 2, pp. 165-168, 2011.

[16] K. Hagberg, E. Häggström, M. Uden, and R. Brånemark, "Socket versus bone-anchored transfemoral prostheses: Hip range of motion and sitting comfort," Prosthet. Orthot. Int., vol. 29, no. 2, pp. 153-163, 2005.

[17] F. Clemente et al., "Touch and Hearing Mediate Osseoperception," Sci. Rep., 2017.

[18] R. Jacobs, R. Brånemark, K. Olmarker, B. Rydevik, D. Van Steenberghe, and P. I. Brånemark, "Evaluation of the psychophysical detection threshold level for vibrotactile and pressure stimulation of prosthetic limbs using bone anchorage or soft tissue support.," Prosthet. Orthot. Int., vol. 24, no. 2, pp. 133-142, 2000. 
[19] H. Van de Meent, M. T. Hopman, and J. P. Frölke, "Walking ability and quality of life in subjects with transfemoral amputation: a comparison of osseointegration with socket prostheses," Arch. Phys. Med. Rehabil., vol. 94, no. 11, pp. 2174-2178, 2013.

[20] M. Al Muderis, W. Lu, and J. J. Li, "Osseointegrated Prosthetic Limb for the treatment of lower limb amputations : Experience and outcomes.," Unfallchirurg, vol. 120, no. 4, pp. 306311, 2017.

[21] M. Al Muderis et al., "The Osseointegration Group of Australia Accelerated Protocol (OGAAP1) for two-stage osseointegrated reconstruction of amputated limbs," Bone Joint J., vol. 98-B, no. 7, pp. 952-960, 2016.

[22] A. Thesleff, E. Haggstrom, R. Tranberg, R. Zugner, A. Palmquist, and M. Ortiz-Catalan, "Loads at the Implant-Prosthesis Interface During Free and Aided Ambulation in Osseointegrated Transfemoral Prostheses," IEEE Trans. Med. Robot. Bionics, 2020.

[23] W. C. C. Lee et al., "Kinetics of transfemoral amputees with osseointegrated fixation performing common activities of daily living," Clin. Biomech., vol. 22, no. 6, pp. 665-673, 2007.

[24] L. Frossard, "Loading characteristics data applied on osseointegrated implant by transfemoral bone-anchored prostheses fitted with basic components during daily activities," Data Br., 2019.

[25] W. Xu, D. H. Xu, and A. D. Crocombe, "Three-Dimensional Finite Element Stress and Strain Analysis of a Transfemoral Osseointegration Implant," Proc. Inst. Mech. Eng. Part H J. Eng. Med., vol. 220, no. 6, pp. 661-670, 2006.

[26] P. K. Tomaszewski, N. Verdonschot, S. K. Bulstra, and G. J. Verkerke, "A comparative finiteelement analysis of bone failure and load transfer of osseointegrated prostheses fixations," Ann. Biomed. Eng., 2010.

[27] P. K. Tomaszewski, M. van Diest, S. K. Bulstra, N. Verdonschot, and G. J. Verkerke, "Numerical analysis of an osseointegrated prosthesis fixation with reduced bone failure risk and periprosthetic bone loss," J. Biomech., vol. 45, no. 11, pp. 1875-1880, 2012.

[28] P. K. Tomaszewski, N. Verdonschot, S. K. Bulstra, J. S. Rietman, and G. J. Verkerke, "Simulated bone remodeling around two types of osseointegrated implants for direct fixation of upperleg prostheses," J. Mech. Behav. Biomed. Mater., 2012.

[29] D. H. Xu, A. D. Crocombe, and W. Xu, "Numerical evaluation of bone remodelling associated with trans-femoral osseointegration implant - A 68 month follow-up study," J. Biomech., vol. 49, no. 3, pp. 488-492, 2016.

[30] M. A. C. Elder, S. Konvickova, M. Daniel, and Z. Horak, "Identification of the critical level of implantation of an osseointegrated prosthesis for above-knee amputees," Computer Methods in Biomechanics and Biomedical Engineering, pp. 1-8, 2017.

[31] P. Prochor and E. Sajewicz, "A Comparative Analysis of Standardised Threads for Use in Implants for Direct Skeletal Attachment of Limb Prosthesis: A Finite Element Analysis," Appl. Bionics Biomech., vol. 2019, no. February, pp. 1-10, 2019.

[32] P. Prochor and E. Sajewicz, "The Influence of Geometry of Implants for Direct Skeletal Attachment of Limb Prosthesis on Rehabilitation Program and Stress-Shielding Intensity," Biomed Res. Int., 2019.

[33] P. Prochor, L. Frossard, and E. Sajewicz, "Effect of the material's stiffness on stress-shielding 
in osseointegrated implants for bone-anchored prostheses: A numerical analysis and initial benchmark data," Acta Bioeng. Biomech., 2020.

[34] W. Xu, a D. Crocombe, and S. C. Hughes, "Finite element analysis of bone stress and strain around a distal osseointegrated implant for prosthetic limb attachment.," Proc. Inst. Mech. Eng. H., vol. 214, no. 6, pp. 595-602, 2000.

[35] W. Lee et al., "Finite element modeling to aid in refining the rehabilitation of amputees using osseointegrated prostheses," Digit. Hum. Model., vol. 4561, pp. 655-658, 2007.

[36] B. Helgason, H. Pálsson, T. P. Rúnarsson, L. Frossard, and M. Viceconti, "Risk of failure during gait for direct skeletal attachment of a femoral prosthesis: A finite element study," Med. Eng. Phys., vol. 31, no. 5, pp. 595-600, 2009.

[37] P. K. Tomaszewski, N. Verdonschot, S. K. Bulstra, and G. J. Verkerke, "A comparative finiteelement analysis of bone failure and load transfer of osseointegrated prostheses fixations," Ann. Biomed. Eng., vol. 38, no. 7, pp. 2418-2427, 2010.

[38] Y. Li and B. Lindeque, "Percutaneous Osseointegrated Prostheses for Transfemoral Amputations," Trending Orthop., vol. 41, no. 2, pp. 75-81, 2018.

[39] P. Stenlund, M. Trobos, J. Lausmaa, R. Brånemark, P. Thomsen, and A. Palmquist, "The effect of load on the bone-anchored amputation prostheses," J. Orthop. Res., vol. 35, no. 5, pp. 1113-1122, 2016.

[40] B. W. Hoyt, S. A. Walsh, and J. A. Forsberg, "Osseointegrated prostheses for the rehabilitation of amputees (OPRA): results and clinical perspective," Expert Rev. Med. Devices, 2020.

[41] A. Palmquist, S. H. Windahl, B. Norlindh, R. Brånemark, and P. Thomsen, "Retrieved boneanchored percutaneous amputation prosthesis showing maintained osseointegration after 11 years-a case report.," Acta Orthop., vol. 85, no. 3, pp. 442-445, 2014.

[42] D. T. Reilly and A. H. Burstein, "The elastic and ultimate properties of compact bone tissue," J. Biomech., vol. 8, no. 6, pp. 393-405, 1975.

[43] P. Zioupos and J. D. Currey, "Changes in the stiffness, strength, and toughness of human cortical bone with age," Bone, 1998.

[44] D. A. Bemben, V. D. Sherk, W. J. J. Ertl, and M. G. Bemben, "Acute bone changes after lower limb amputation resulting from traumatic injury," Osteoporos. Int., vol. 28, no. 7, 2017.

[45] V. D. Sherk, M. G. Bemben, and D. A. Bemben, "BMD and Bone Geometry in Transtibial and Transfemoral Amputees," J. Bone Miner. Res., vol. 23, no. 9, pp. 1449-1457, 2008.

[46] X. N. Dong and X. E. Guo, "The dependence of transversely isotropic elasticity of human femoral cortical bone on porosity," J. Biomech., 2004.

[47] N. E. Dowling, Mechanical behavior of materials: Engineering Methods for Deformation, Fracture and Fatigue, Third Edition, vol. 46, no. 12. 2007.

[48] J. E. Shigley, C. R. Mischke, and R. G. Budynas, Shigley's Mechanical Engineering Design - 9th Ed., vol. New York, 2002.

[49] U. Wolfram and J. Schwiedrzik, "Post-yield and failure properties of cortical bone," Bonekey Rep., 2016.

[50] C. T. Rubin and L. E. Lanyon, "Regulation of bone mass by mechanical strain magnitude," Calcif. Tissue Int., 1985. 
